NASA Technical Memorandum 105332 ICOMP -91-23

\title{
Euler Solutions for an Unbladed Jet Engine Configuration
}

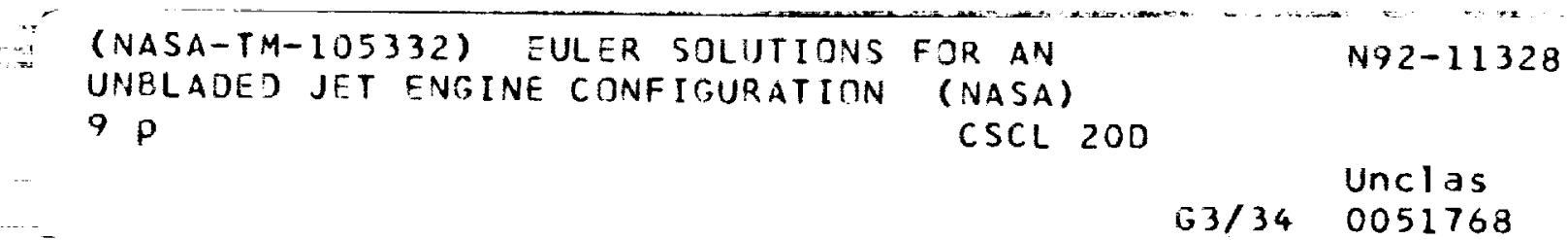

Mark E.M. Stewart

Institute for Computational Mechanics in Propulsion

Lewis Research Center

Cleveland, Ohio

Prepared for the

30th Aerospace Sciences Meeting and Exhibit sponsored by the American Institute of Aeronautics and Astronautics Reno, Nevada, January 6-9, 1992







\title{
EULER SOLUTIONS FOR AN UNBLADED JET ENGINE CONFIGURATION
}

\author{
Mark E. M. Stewart \\ Institute for Computational Mechanics in Propulsion \\ NASA Lewis Research Center \\ Cleveland, Ohio
}

\begin{abstract}
An Euler solution for an axisymmetric jet engine configuration without blade effects is presented. The Euler equations are solved on a multiblock grid which covers a domain including the inlet, bypass duct, core passage, nozzle, and the far field surrounding the engine. The simulation is verified by considering five theoretical properties of the solution. The solution demonstrates both multiblock grid generation techniques and a foundation for a full jet engine throughflow calculation.
\end{abstract}

\section{Introduction}

Numerical simulations increasingly realize the hope of simulating a wide range of physical processes for engineering design and scientific research. One facet of realizing this goal is developing numerical simulations for geometrically complex domains. Another facet is developing numerical techniques appropriate to a problem of interest. Thus the simulation of an engine configuration is both a testbed for simulations for complex geometries and provides a tool for engine research and design.

A central issue in performing simulations in complex geometries is determining the grid: the choice of points on which the equations will be discretized and how these points are organized to interact with the numerical solution techniques. There are many specific approaches to this problem, but the grids are either locally structured or unstructured. Structured grids have a trivial local relationship between neighbors while unstructured grids have neighbor relationships stored in tables. This difference has implications for the algorithms which may be implemented, the methods of generating grids and ancillary issues. For example, matrix formulations of implicit schemes on unstructured grids lose the bandedness possible with structured grids. However, when using structured grids, the burden of dealing with complex geometries shifts to how the blocks interact with each other.

For these classes of grids, there are many approaches to complex geometry simulations. Euler solutions for aircraft configurations using unstructured grids have been presented by Jameson, Baker, and Weatherill ${ }^{1}$ and

This paper is declared a work of the U.S. Government and is not subject to copyright protection in the United States
Lohner and Parikh. ${ }^{2}$ Buning et. al. $^{3}$ have simulated an ascending Space Shuttle using overset grids, and Chesshire and Henshaw ${ }^{4}$ have demonstrated NavierStokes solutions with overlapping grids in two dimensions. Non-overlapping, structured multiblock grids and solutions for aircraft configurations have been used by Sawada and Takanashi ${ }^{5}$ while Whitfield et. al. ${ }^{6}$ have found Euler solutions for counter-rotating propfans where the structured grids are in relative motion. Hall et. al. ${ }^{7}$ have demonstrated solutions for a ducted prop-fan. Stewart ${ }^{8}$ has used multiblock grids to find Euler solutions for multi-element airfoil sections.

However, for multi-stage turbomachinery, significant geometrical and physical complications are added to a simulation. Blade rows are in relative motion and interact strongly so simplifications and physical modelling are still necessary. One pioneering approach to this problem was proposed by $\mathrm{Wu}^{9}$, and it represents the flow through relatively moving blade rows as two distortable streamsurfaces. Jennions and Stowe $\mathrm{e}^{10,11}$ have shown quasi-three dimensional throughflow calculations for a turbine stage. Wisler, Koch, and Smith ${ }^{12}$ mention the use of circumferential average flow calculations in preliminary compressor design. More recently, averaging models ${ }^{13,14,15}$ have been proposed to simulate three dimensional multi-stage turbomachines.

Although component performance is important, there are interactions between compressors, combustors, and other components. After initial analysis and experimental testing of an engine design, components are integrated based on their performance in isolation. Thus consideration of engine integration effects is limited to one dimensional analysis and final engine testing, even when less conservative designs require consideration of these interaction effects.

The purpose of the current work is to lay a foundation for modelling some of these integration effects. As a step in this task, the current calculation deals with the geometrical and numerical problems of combined internal and external flow for an engine configuration. Section 2 details the geometry modelling and motivates the choices and changes which have been made. Section 3 summarizes the techniques used to create the multiblock grids, and Section 4 explains the numerical methods employed. The numerical results are presented in Section 5. 


\section{Geometry Modelling}

The geometry of the simulated engine configuration is based on the General Electric Energy Efficient Engine $\left(E^{3}\right)$. The geometry is specifically based on the Integrated Core/Low Spool (ICLS) ground test configuration for which there is extensive test data. ${ }^{16}$ Development of the $E^{3}$ engine was sponsored by NASA as part of a broad-based effort to increase aircraft efficiency.

Several modifications have been made to this geometry to achieve the desired configuration and to allow for a convergent solution. No attempt has been made to model the combustor of the engine at this stage of the simulation, and a duct has been installed from the compressor to the turbine section. A small booster island has been excluded. Further, because the ICLS ground test did not include the external flowpath of the nacelle, one has been designed for this case. An ellipse is used from the throat of the inlet to the highlight. The external nacelle contour is a NACA 1-Series forebody and the aft section is an arbitrarily-fit Bezier spline. In the future, improved geometrical descriptions may be readily incorporated into the simulation.

The surfaces are modelled using splines parameterized by arc length. To ensure the integrity of this representation, continuous first derivatives of the raw data are ensured wherever possible. Also, it has been necessary to smooth rough surface data in the compressor and turbine sections. A least squares fit to a basis set of sine, cosine functions was successful in removing these high frequency geometrical irregularities.

In the current calculations, blade effects have been neglected while the geometrical issues of throughflow calculations are studied. Without blade effects to do work and extract energy from the stream, the flow through the core section is significantly changed. In particular, the compressor blades do no work to assist the flow through the core section and achieve the 23:1 pressure ratio of the engine's compressor. It was necessary to change the core surface contour so that the maximum to minimum area through the core section is approximately $3: 1$. This change was achieved by both lifting the entire splitter and altering its core contour.

\section{Grid Generation Techniques}

The grid for this engine configuration is developed using the TOPOS program. ${ }^{8,17}$ This program determines a domain decomposition such that the domain is covered with non-overlapping regions which are each topologically rectangular and hence called blocks. Each block is dimensioned so that it contains a structured grid while the block interfaces have coordinate lines continue through them without slope discontinuities.

The domain decomposition is performed using a search algorithm which finds boundary conforming regions in a two-dimensional domain. ${ }^{17}$ In the same way that the skin of a balloon will conform to the bounding walls when blown up in a confined space, this algorithm refines a coarse approximation to the perimeter of a region so that it conforms to any nearby neighboring boundaries without excessive stretching.

This algorithm is demonstrated in figure 1 by considering the simple case of an airfoil in a box, $P Q R S$, and an initial, coarse approximation to the region below the airfoil, $A C R S$. To transform $A C R S$ to a region which conforms to the lower surface of the airfoil, the algorithm finds the point which determines the highest flat ceiling above the middle third of $A B, E F$, as in figure 1b). Limiting the depth of this probe to the height Search Depth prevents finding points on the segment $P Q$ which would yield a perimeter with excessive stretching. The probe considers the curves which define the domain and finds the point $D$. The perimeter is then modified to $A D B C$, and two child segments, $A D$ and $D B$, are created from $A B$.

a)
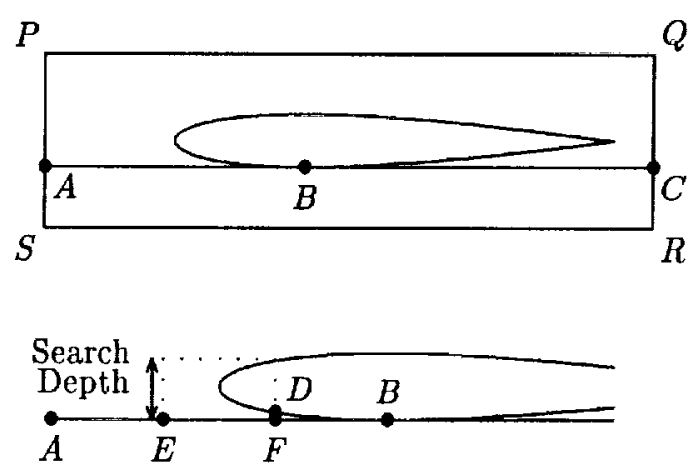

b)

c)
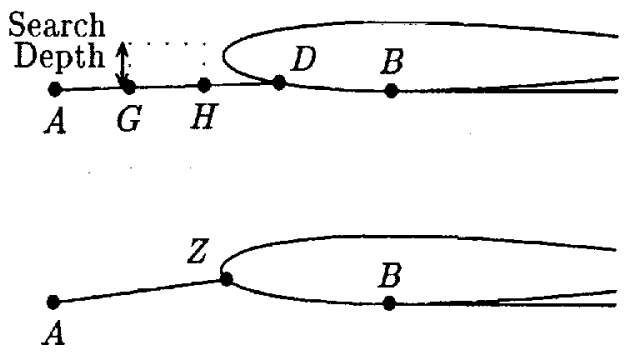

d)

Figure 1: Steps in Finding a Boundary Conforming Region.

The refinement of $A D B$ involves refining $A D$ and $D B$. $A D$ is refined, but $D B$ is not since it is part of a boundary defining curve. Probing above the middle third of $A D, G H$, yields no point within search depth so the two outer segments $A G$ and $H D$ are considered for refinement. This failure to find a highest ceiling implies there will be a transition between bodies in the perimeter. This refinement is extended in a tree structure by considering successively smaller sub-segments as above, and eventually yields the perimeter $A Z B$ of figure 1d). 
With this algorithm, boundary conforming regions are successively found and removed until the domain is covered.

After finding a decomposition, transformations may be applied to it which involve cutting and merging blocks in general ways. The data structures which describe the decomposition are designed in such a way that these transformations are isomorphic with respect to the data structures, providing a tool for the development and adaptation of multiblock grids.

Given a domain decomposition, it is necessary to determine the dimensions of the grids in each block. To have a structured grid with coordinate lines continuous through the block interfaces, two conditions must be satisfied. First, the numbers of cells on opposite sides of a block must be equal; second, the number of cells on opposite sides of a block interface must be equal. These conditions may be expressed as an underconstrained system of linear, integer coefficient equations with integer solutions. If the equations have an admissible solution, a linear programming algorithm will find the solution. Further, the free parameters in the solution are also found, providing a technique for global adaptation of the grid.

To obtain grids within the blocks, elliptic grid generation $^{18}$ is used to smooth initial approximations to the grid found by algebraic methods. The interfaces between blocks are also smoothed. Having a locally smooth grid which may be thought of as a locally $\mathrm{C}^{1}$ transformation between physical and computational space results in a second order local discretization error in the numerical approximation, as will be explained in Section 4.

\section{Numerical Methods}

The two-dimensional Euler equations model inviscid, compressible, rotational flow and allow entropy and vorticity production across shocks. For future applications, they will allow for body forces and energy addition. The Euler equations are given by

$$
\frac{d}{d t} \int_{\Omega} w d \mathrm{Vol}=-\oint_{\partial \Omega} F \cdot n d s
$$

where $F=(f, g)$ and

$$
\begin{gathered}
w=\left(\begin{array}{c}
\rho \\
\rho u \\
\rho v \\
\rho E
\end{array}\right) \\
f(w)=\left(\begin{array}{c}
\rho u \\
\rho u^{2}+P \\
\rho u v \\
\rho u H
\end{array}\right) \quad g(w)=\left(\begin{array}{c}
\rho v \\
\rho u v \\
\rho v^{2}+P \\
\rho v H
\end{array}\right)
\end{gathered}
$$

$$
P=(\gamma-1)\left(\rho E-\frac{\rho\left(u^{2}+v^{2}\right)}{2}\right) \quad H=\frac{\gamma}{(\gamma-1)} \frac{P}{\rho}
$$

Here $\gamma=1.4$. The circumferential velocity is assumed to be identically zero.

The numerical approximation and solution techniques are based on the FLO52 program of Jameson et. al. ${ }^{19}$ The equations are discretized by taking the dependent variables, $w$, to be located at the centroids of the grid cells. The convective flux for a cell, $Q(w)$, is approximated from the average dependent variable value between adjacent cells and the area of the cell face.

The discretized equations for each cell are advanced to a steady state by a multi-stage scheme:

$$
\begin{gathered}
w^{(1)}=w_{i}+\alpha_{1} \Delta t\left(Q\left(w_{i}\right)+D\left(w_{i}\right)\right) \\
w^{(2)}=w_{i}+\alpha_{2} \Delta t\left(Q\left(w^{(1)}\right)+D\left(w^{(1)}\right)\right) \\
w^{(3)}=w_{i}+\alpha_{3} \Delta t\left(Q\left(w^{(2)}\right)+D\left(w^{(1)}\right)\right) \\
w^{(4)}=w_{i}+\alpha_{4} \Delta t\left(Q\left(w^{(3)}\right)+D\left(w^{(1)}\right)\right) \\
w_{i+1}=w_{i}+\alpha_{5} \Delta t\left(Q\left(w^{(4)}\right)+D\left(w^{(1)}\right)\right)
\end{gathered}
$$

where $\alpha_{i}$ are coefficients, $Q(w)$ is the convective flux approximation for the cell, and $D(w)$ is the artificial dissipation. The artificial dissipation consists of third-order dissipation, which stabilizes the time stepping scheme, and first-order dissipation, which is switched on near shocks to capture them.

Neumann stability analysis of Equation (2) for a onedimensional analog of Equation (1) indicates that stability is expected for appropriate coefficients $\alpha_{i}$. Since a steady state solution is sought, convergence is accelerated by using the maximum time step in each cell. For isenthalpic conditions, convergence may be enhanced by using enthalpy damping. ${ }^{20}$ Enthalpy damping uses the Total Energy, $E$, and kinetic energy, $\frac{1}{2}\left(u^{2}+v^{2}\right)$, to estimate the local deviation from constant enthalpy and force the mass and momentum equations accordingly. In the absence of body forces and energy release, enthalpy damping has been used.

Convergence has also been enhanced by using a multigrid algorithm. ${ }^{21}$ Inset grids are included by having each grid dimension contain a factor of a power of two. The number of multigrid levels is limited by the coarsest unstretched grid which in turn is determined by the shortest block side. For this calculation, three levels can be constructed, and their use improves convergence considerably. The rate of convergence, measured by the average log residual reduction per cycle, doubles for each added level.

At solid boundaries, a free slip condition is applied and pressure is extrapolated to the surface by considering grid skew and centrifugal effects. At the boundary in the far field, a uniform free stream velocity is assumed 


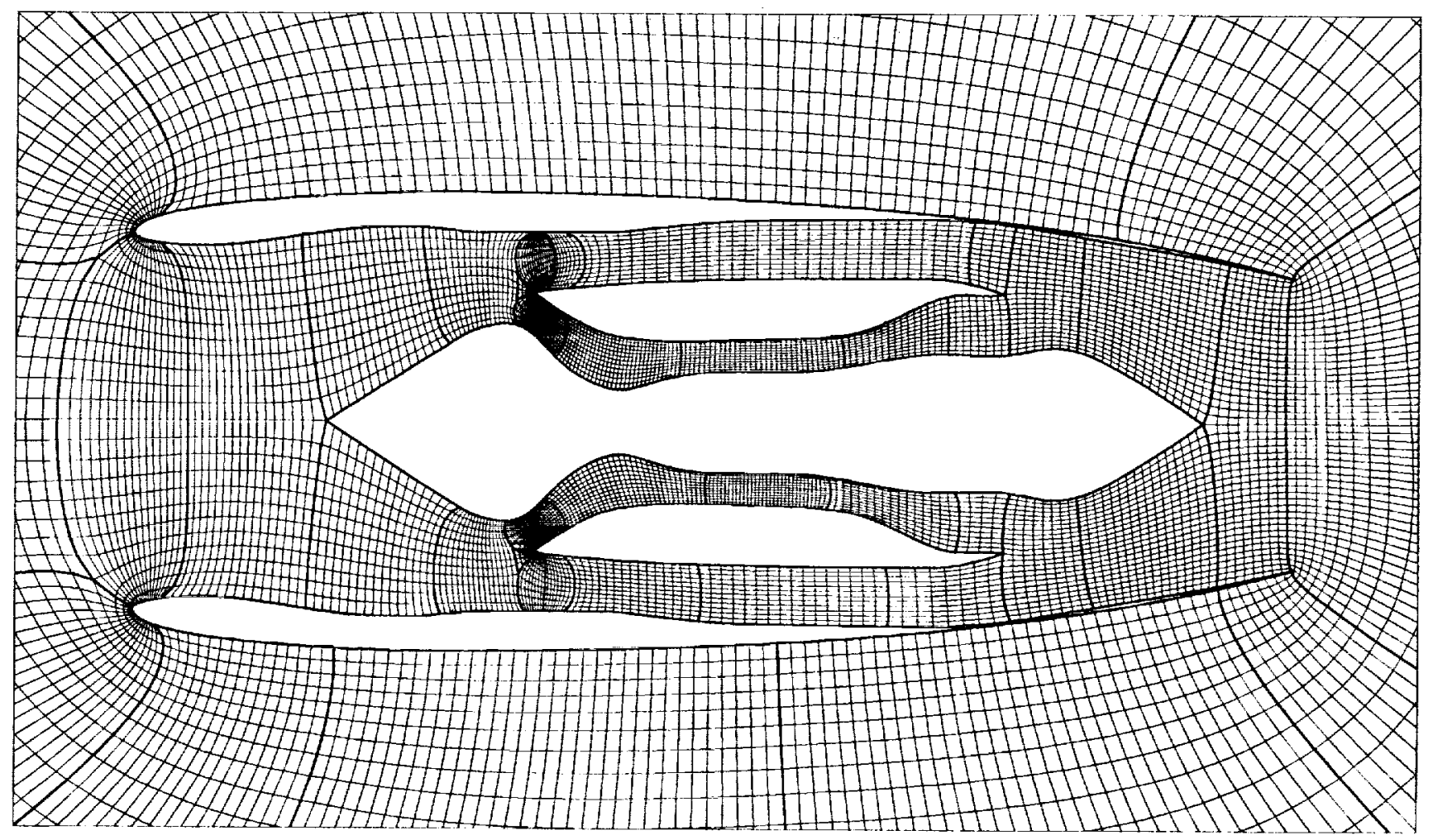

Figure 2: Inner Region of the Grid for the Jet Engine Configuration

and matched to the solution using Riemann invariants. At interfaces between blocks, a simple matching of the solutions is possible. Since coordinate lines are continuous through the interface, the grid may be continued across a block interface. Consequently, the dependent variable values are known for the flux approximation, $Q(w)$, and artificial dissipation, $D(w)$ at each interface. Because communication occurs between grids by copying data values at each stage of Equation (2), interpolation is unnecessary. Further, these conditions result in the property that splitting blocks does not change the solution or convergence--they are identical in both cases. The only penalties paid for splitting blocks are for setting up additional loops and subroutine calls and for decreased vector lengths.

Since coordinate lines are $\mathrm{C}^{1}$ through an interface, it is possible to make theoretical arguments that the solution is locally second order accurate. ${ }^{17}$ The truncation error of the flux approximation, $Q(w)$, may be expressed on the locally uniform computational domain because there is a local $\mathrm{C}^{1}$ transformation between physical and computational space. In this form, it can be shown that the truncation error should be second order. This result has been numerically verified on grids of this type. This conclusion is also supported by examination of the drag coefficient computed on grids of varying resolution.

\section{Results}

The geometrical domain includes the contours for the hub, splitter, and nacelle which are symmetric about the engine axis, and extends 13 engine lengths into the far field to a circular boundary of truncation. The grid for the domain, shown in figure 2, contains 16800 cells in 35 blocks whose edges are indicated by bold lines.

The grid contains three inset multigrid levels which are used to enhance convergence. These grid levels are created from a coarse base grid by multiplying each grid dimension by a factor of four. Additional grid levels would enhance convergence further, but using a coarser base grid with this non-uniform grid topology would result in excessive grid stretching and degrade the accuracy of the solution.

Although the geometry is symmetric about the centerline, the grid is not. The grids in the upper and lower half planes are generated without a symmetry condition applied between the two halves, except that the engine axis corresponds to a coordinate line. This grid asymmetry is exploited to verify the solution, since the solution will not be symmetric because of the grid but because of proper resolution. Because the coordinate line on the axis does not necessarily correspond to a block interface, some blocks are in both the upper and lower half planes. Having the centerline correspond to 


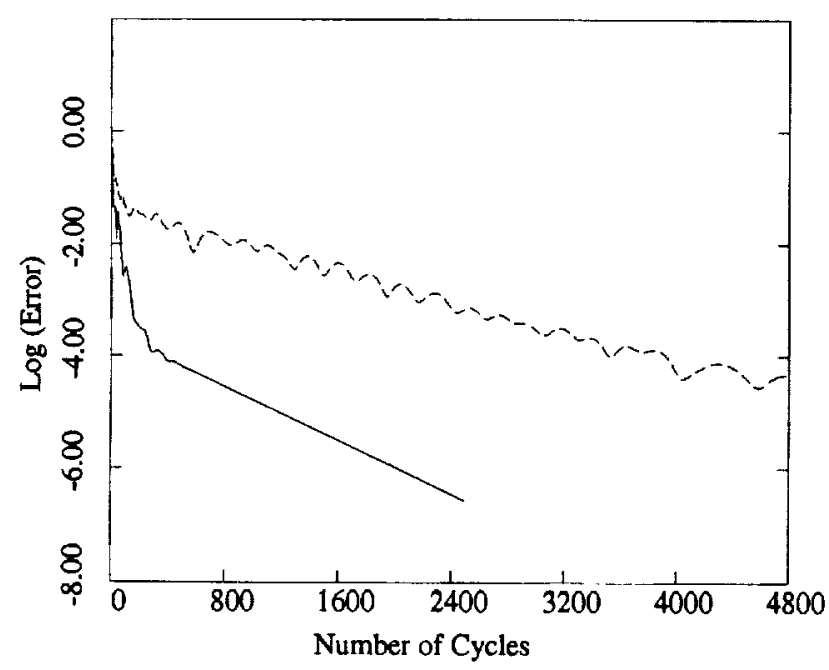

Figure 3: Convergence Histories with Two Multigrid Levels (solid line) and Without Multigrid (dashed line)

the engine axis results in a condition of zero flux through the axis, which isolates the two half planes. There is also circumferential isolation since the grid does not extend in the third dimension, theta.

The Euler solution was run for 2500 cycles on this grid at Mach $M=0.3$ and an angle of attack $\alpha=0^{\circ}$. The Mach number, $M=0.3$, was chosen for this case to avoid transonic flow and choking in the engine core passage. Since no work is done by the blades on the fluid through the compressor section, the fluid accelerates dramatically and can become sonic only at the throat of the duct. This sonic point limits the mass flow through the duct and hence the capture streamline for the core. A low Mach number was chosen to minimize this problem.

Although the ground test data ${ }^{16}$ is not an appropriate basis for comparison at this stage because no blade effects are being considered, the results may be verified based on five theoretical properties of the solution. First, the solution is convergent in the sense that mass is conserved locally. The density residual, $\frac{d \rho}{d t}$, RMS averaged over the field, is shown in figure 3 , and indicates convergence. This quantity is a direct measure of how close to mass conservation the solution is. The energy equation is also convergent. Convergence of the total energy residual, $\frac{d \rho E}{d t}$, over the field is a direct measure of how close to energy conservation the solution is.

Second, since the solution is subcritical, it should be isentropic, and deviations are an indication of error. The deviation from constant entropy over the surfaces is shown in figure 4. The leading edge of the nacelle contributes the largest amount to the entropy deviations in part because the limited ingestion of air into the engine inlet moves the capture streamline closer to the engine axis and the stagnation point into the inlet. As a result, substantial turning of the flow around the leading edge of the nacelle is required. The leading edge
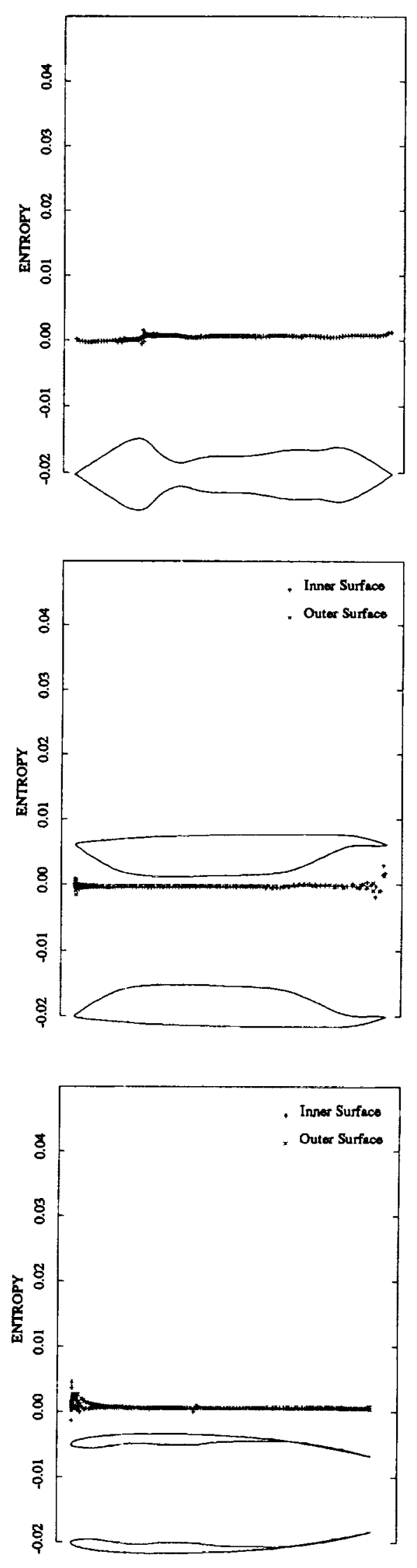

Figure 4: Fractional Deviation of Entropy From its Free Stream Value Over the Surfaces of the Configuration 

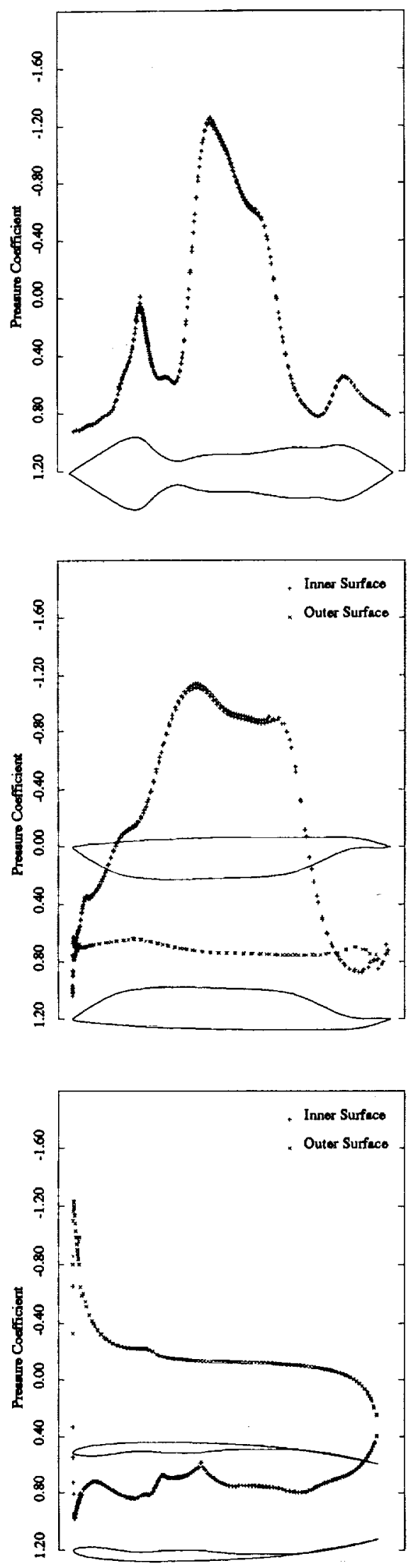

Figure 5: Pressure Distributions Over the Symmetric Surfaces of the Configuration of the splitter plate is difficult to resolve because it has a small leading-edge radius, which creates an entropy deviation.

Third, by geometrical symmetry, the lift coefficient, $C_{l}$, should theoretically be zero, and on a symmetric grid in symmetric conditions, one would expect the numerical result to be identically zero due to cancellation. However, for a symmetric geometry and an asymmetric grid, one cannot expect an identically zero value. The computed lift coefficient is $C_{l}=-0.0009$ and is due to grid asymmetry, the truncation errors in the numerical approximations, and artificial dissipation.

Fourth, since the Euler solution is sub-critical, the drag coefficient, $C_{d}$, theoretically should be zero. The value $C_{d}=0.0009$ can again be attributed to grid asymmetry, errors due to numerical approximations, and artificial dissipation.

The symmetry of the geometry and the asymmetry of the grid provide a fifth test. The surface distributions of flow quantities should be symmetric. The surface pressure distributions are sensitive to errors and the distributions for the three symmetric components are shown in figure 5 .

\section{Conclusions}

A solution to the Euler equations for an axisymmetric jet engine configuration without blade effects has been presented. The solution has been found on a multiblock grid which includes both the internal ducts and the external flow surrounding the engine. Further, the result has been verified on the basis of five theoretical properties of the solution. Thus this work demonstrates techniques for finding numerical solutions in complex geometries and provides a foundation for more detailed modelling of engine components.

\section{Acknowledgements}

I would like to thank John Abbott for guidance in the external nacelle design, Chuck Putt for help with the geometry specification, and Andy Kuchar of General Electric for providing the hot flowpath coordinates for the Energy Efficient Engine ICLS configuration. Gregory Swartwout has kept the computers running without fail. The Euler solver is based on the FLO52 program of Antony Jameson. I would also like to thank Jeff Yokota and John Adamczyk for valuable discussions and advice, and John for suggesting this problem.

\section{References}

1 Jameson, A., Baker, T. J., and Weatherill, N. P., "Calculation of Inviscid Transonic Flow over a Com plete Aircraft," AIAA Paper 86-0103, Jan. 1986.

2 Lohner, R. and Parikh; P., "Generation of Three- 
Dimensional Unstructured Grids by the AdvancingFront Method," AIAA Paper 88-0515, Jan. 1988.

${ }^{3}$ Buning, P., Chiu, I., Obayashi, S., Rizk, Y. and Steger, J., "Numerical Simulation of the Integrated Space Shuttle Vehicle in Ascent," AIAA Paper 884359, Aug. 1988

4 Chesshire, G. and Henshaw, W., "Composite Overlapping Meshes for the Solution of Partial Differential Equations," Journal of Computational Physics, Vol. 90, 1990, pp. 1-64.

5 Sawada, K. and Takanashi, S., "A Numerical Investigation on Wing/Nacelle Interferences of USB Configuration," AIAA Paper 87-0455 Jan. 1987.

6 Whitfield, D., Swafford, T., Mulac, R., Belk, D. and Janus, J., "Three-dimensional Unsteady Euler Solutions for Propfans and Counter-rotating Propfans in Transonic Flow," AIAA Paper 87-1197 June 1987.

7 Hall, E., Delaney, R. and Bettner, J., "Investigation of Advanced Counterrotation Blade Configuration Concepts for High Speed Turboprop Systems - Task 1 Ducted Propfan Analysis," NASA CR - 185217, April 1990.

${ }^{8}$ Stewart, M., "A General Decomposition Algorithm Applied to Multi-Element Airfoil Grids," AIAA Paper 90-1606, Seattle, June 1990.

9 Wu, C. H., "A General Theory of Three-Dimensional Flow in Subsonic or Supersonic Turbomachines of Axial-, Radial-, and Mixed-Flow type," NACA TN 2604, 1952

10 Jennions, I. and Stowe, P., "A Quasi-Three-Dimensional Turbomachinery Blade Design System: Part I- Throughflow Analysis," ASME Journal of Engineering for Gas Turbines and Power, Vol. 107 April 1985 pp. 301-307.

11 Jennions, I. and Stowe, P., "A Quasi-Three-Dimensional Turbomachinery Blade Design System: Part II - Computerized System," ASME Journal of Engineering for Gas Turbines and Power, Vol. 107 April 1985 pp. 308-316.

12 Wisler, D., Koch, C. and Smith, L., "Preliminary Design Study of Advanced Multistage Axial Flow Core Compressors," NASA CR-135133, Feb. 1977.

13 Dawes, W. N., "Towards Improved Throughflow Capability - The Use of 3D Viscous Flow Solvers in a Multistage Environment," ASME Paper 90GT-18, June 1990.

14 Denton, J. D., "The Calculation of Three Dimensional Viscous Flow through Multistage Turbomachines," ASME Paper 90-GT-19, June 1990.

I5 Adamczyk, J., Celestina, M., Beach, T. and Barnett, M., "Simulation of 3-D Viscous Flow Within a Multi-Stage Turbine," NASA TM-101376 June, 1989.

16 Stearns, E. M., "Energy Efficient Engine Integrated Core/Low Spool Design and Performance Report," NASA CR-168211, Feb. 1985.
17 Stewart, M., "Non-Overlapping Multiblock Meshes for Multi-Element Airfoils with Euler Solutions," $\mathrm{Ph}$. D. Thesis, Program in Applied and Computational Mathematics, Princeton University, June 1990.

18 Thompson, J., Thames, F. and Mastin, C., "Automatic Numerical Generation of Body-Fitted Curvilinear Coordinate System for Field Containing Any Number of Arbitrary Two-Dimensional Bodies," Journal of Computational Physics, Vol. 15, 1974, pp. 299-319.

19 Jameson, A., Schmidt, W. and Turkel, E., "Numerical Solution of the Euler Equations by Finite Volume Methods Using Runge-Kutta Time Stepping Schemes," AIAA Paper 81-1259, June 1981.

20 Jameson, A., "Steady State Solutions of the Euler Equations for Transonic Flow," Transonic, Shock and Multidimensional Flows: Advances in Scientific Computing, Academic Press, N.Y. 1982.

21 Jameson, A., "Solution of the Euler Equations for Two Dimensional Transonic Flow by a Multigrid Method," Applied Mathematics and Computation, Vol. 13 1983, pp 327-356. 
Public reporting burden for this collection of information is estimated to average 1 hour per response, including the time for revlewing instructions, searching existing data sources, gathering and maintaining the data needed, and completing and reviewing the collection of information. Send comments regarding this burden estimate or any other aspect of this collection of Inlormalton, including suggestions for reducing this burden, to Washington Headquarters Services, Directorate lor inlormation Operations and Reports, 1215 Jefterson Davis Highway, Suite 1204, Arlinglon, VA 22202-4302, and to the Otfice of Management and Budget, Papenwork Peduction Project (0704-0168), Washinglon, DC 20503.

1. AGENCY USE ONLY (Leave blank)

\section{TITLE AND SUBTITLE}

Euler Solutions for an Unbladed

Jet Engine Configuration

\section{AUTHOR(S)}

Mark E.M. Stewart

\section{REPORT TYPE AND DATES COVERED}

Technical Memorandum

\section{PERFORMING ORGANIZATION NAME(S) AND ADDRESS(ES)}

National Aeronautics and Space Administration

Lewis Research Center

Cleveland, Ohio 44135-3191
WU-505-62-2I

B. PERForming ORgANIZATION REPORT NUMBER

$E-6695$

\section{SPONSORING/MONITORING AGENCY NAMES(S) AND ADDRESS(ES)}

National Aeronautics and Space Administration

Washington, D.C. 20546-0001
10. SPONSORINGMONITORING AGENCY REPORT NUMBER

NASA TM -105332

ICOMP - 91-23

\section{SUPPLEMENTARY NOTES}

Prepared for the 30th Aerospace Sciences Meeting and Exhibit sponsored by the American Institute of Aeronautics and Astronautics, Reno, Nevada, January 6-9, 1992. Mark E.M. Stewart, Institute for Computational Mechanics in Propulsion, Lewis Research Center (work funded under Space Act Agreement C-99066-G). Space Act Monitor: Louis A. Povinelli, (216) 433-5818.

12. DISTRIBUTION/AVAILABILITY STATEMENT 12b. DISTRIBUTION CODE

Unclassified - Unlimited

Subject Category 34

\section{ABSTRACT (Maximum 200 words)}

An Euler solution for an axisymmetric jet engine configuration without blade effects is presented. The Euler equations are solved on a multiblock grid which covers a domain including the inlet, bypass duct, core passage, nozzle, and the far field surrounding the engine. The simulation is verified by considering five theoretical properties of the solution. The solution demonstrates both multiblock grid generation techniques and a foundation for a full jet engine throughflow calculation.

14. SUBJECT TERMS

Computational fluid dynamics; Grid generations; Jet engines; Euler solutions

15. NUMBER OF PAGES

8

16. PRICE CODE

$\mathrm{A02}$

17. SECURITY CLASSIFICATION
OF REPORT
Unclassified

18. SECUAITY CLASSIFICATION OF THIS PAOE

Unclassified

NSN 7540-01-280-5500
19. SECURITY CLASSIFICATION OF ABSTRACT Unclassified 